\title{
Associação de métodos fisioterapêuticos para o tratamento da doença metacarpiana dorsal em eqüinos
}

\author{
Treatment of equine dorsal metacarpal disease using physiotherapeutic methods \\ Simone Isler Ferraz Bravo Caldeira ${ }^{1}$ José Roberto Cintra do Prado-Filho ${ }^{1}$ \\ Raquel Yvonne Arantes Baccarin ${ }^{2 *}$
}

\section{RESUMO}

\begin{abstract}
A periostite do terceiro metacarpiano acomete 70 a $80 \%$ dos cavalos de corrida em seu primeiro ano de treinamento, levando-os à claudicação e interrupção temporária do trabalho. O objetivo deste artigo consistiu em avaliar um tratamento não farmacológico e não invasivo que diminuísse o período de recuperação e de reparação tecidual. Foram tratados 13 eqüinos Puro Sangue Inglês de corrida (24 membros torácicos), com idade entre 2 e 3 anos, acometidos por periostite do terceiro metacarpiano, e avaliados por 30 dias consecutivos. A fisioterapia incluiu crioterapia, fototerapia, eletroterapia e fonoterapia. O exame físico evidenciou rápida redução do grau de dor, do calor local e do grau de claudicação. Após sete dias de tratamento, 71\% dos animais não mais apresentavam sinais de claudicação. Os exames radiográfico e ultra-sonográfico permitiram analisar a reparação tecidual. Os resultados mostraram os efeitos analgésico e antiinflamatório dos recursos utilizados, permitindo a manutenção do exercício e o retorno rápido ao treinamento, além do reparo tecidual. O protocolo fisioterapêutico proposto favorece sua aplicação em medicina eqüina.
\end{abstract}

Palavras-chave: doença metacarpiana dorsal, eqüino, fisioterapia.

\section{ABSTRACT}

Dorsal metacarpal disease affects 70 to $80 \%$ of thoroughbreds during the first year of training, causing lameness and interruption of training. The aim of this study was to evaluate the efficacy of a non-pharmacological, non-invasive treatment that could shorten the recovery period and healing time. Thirteen thoroughbreds (24 limbs) diagnosed with dorsal metacarpal disease were treated and evaluated during a period of 30 days. The physical therapy consisted of cryotherapy, photon therapy, electrical stimulation and ultrasound therapy. There was prompt reduction in pain, local heat and degree of lameness. After 7 days of treatment, $71 \%$ of the cases showed no lameness. Radiography and ultrasonography were employed to evaluate healing. Results showed significant antiinflammatory and analgesic effects with faster return to training. The physiotherapeutic protocol proposed favors its applicability in equine medicine.

Key words: dorsal metacarpal disease, horse, physical therapy.

\section{INTRODUÇÃO}

A fisioterapia pode ser empregada no tratamento da maioria dos casos que envolvem o sistema locomotor do eqüino, sendo de grande valia na reabilitação de atletas. Atua localmente utilizando técnicas manuais e aparelhos emissores de ondas que permitem a otimização da reparação tecidual por incremento do metabolismo e da osteogênese (LIPPIELLO \& SMALLEY, 1991), a adequação da circulação ao momento inflamatório (PORTER, 1998), a modulação da reação inflamatória e o controle da dor (IVERS, 1987; REDDY, 2004). Apresenta caráter não invasivo, podendo ser utilizada nos casos de periostite do osso terceiro metacarpiano (MCIII), que se manifesta por inflamação subcutânea considerável, resposta periostal das faces dorsal e dorsomedial do terceiro metacarpiano e tendência à cronicidade.

Contudo, foram encontrados somente dois relatos na literatura (LANG, 1980; MONTGOMERY,

\footnotetext{
${ }^{1}$ Faculdade de Medicina Veterinária e Zootecnia (FMVZ), Universidade de São Paulo (USP), São Paulo, São Paulo, Brasil.

${ }^{2}$ Departamento de Clínica Médica da FMVZ, USP. Av. Orlando Marques de Paiva, 87, Butantã, 05508-000, São Paulo, São Paulo, Brasil. E-mail baccarin@usp.br. Autor para correspondência.
} 
1981) referentes à utilização de modalidades fisioterapêuticas no tratamento da periostite do MCIII em eqüinos. BECK (1998), em revisão bibliográfica sobre enfermidade similar em humanos, citou um estudo envolvendo a utilização de estimulação elétrica, que evidenciou redução significativa no tempo de recuperação.

NORWOOD \& HAYNES (1982), com base em pesquisas que relacionam remodelagem óssea com potenciais elétricos em seres humanos, propuseram a utilização de estímulo elétrico para o tratamento da periostite e de fraturas por estresse do MCIII. Além dos efeitos que promovem analgesia e redução dos demais sinais da inflamação, estudos em animais vêm mostrando que várias formas de estimulação elétrica afetam positivamente o crescimento, reparo e remodelamento de tecidos moles e duros (MIKHAILOVA\& LANDA, 1981).

A periostite do MCIII e as fraturas por estresse (fadiga) são manifestações agudas e crônicas do excesso de tensão aplicada sobre o córtex dorsal do MCIII em eqüinos, ocorrendo normalmente quando potros são exercitados a velocidades acima de $12 \mathrm{~ms}^{-1}$ ou no momento da saída do partidor (DAVIES, 1996).

BERTONE (2002) classificou a periostite do MCIII em três tipos, sendo do tipo aguda as que ocorrem em cavalos em início de treinamento, entre oito e 36 meses, e, ocasionalmente, nos de mais idade que não tenham iniciado o treinamento aos dois anos. Tem início agudo, com sinais de dor intensa à pressão. Radiografias nesta fase freqüentemente não monstram alterações ósseas; mesmo assim, às vezes são encontradas fraturas, como também áreas mínimas de osteólise cortical superficial. As lesões do tipo subaguda ou crônica ocorrem em cavalos de aproximadamente 26 a 42 meses. Observa-se apenas grau discreto de irregularidade no andamento, resultante de um tipo agudo não responsivo à terapia ou que passou despercebido. Pela palpação, a reação de dor é variável, sendo mais nítida após exercício. $\mathrm{O}$ aumento de volume principalmente na face dorsomedial é evidente, e a imagem radiográfica é de um calo subperiostal. Por fim, as do terceiro tipo resultam de fratura na face dorsal ou dorsolateral do córtex e são normalmente observadas em cavalos mais velhos, entre três e cinco anos. A presença de dor é evidente após exercício forte. A palpação revela uma discreta área dolorida na superfície dorsolateral do MCIII esquerdo, entre os terços médio e distal. Radiografias normalmente mostram uma fratura cortical da face dorsolateral.

As lesões agudas respondem favoravelmente ao repouso ou mesmo à redução da intensidade do exercício. Porém, mesmo com a associação do repouso, da administração de antiinflamatório não esteróide e de exercício controlado, o eqüino pode ficar em convalescença por períodos maiores. Já as lesões subagudas ou crônicas são mais difíceis de serem tratadas, necessitam de um período maior de repouso, geralmente 110 dias, podendo levar até um ano para a resolução completa. As fraturas corticais dorsais podem ser curadas seguindo-se o recomendado para as lesões agudas; porém, os períodos de convalescença podem se prolongar por quatro a seis meses. Quando não houver resposta ao tratamento conservativo, a indicação terapêutica é cirurgia(SPEIRS, 1994; BERTONE, 2002).

Outros métodos de tratamento incluem utilização de bolsas de gelo, antiinflamatórios tópicos, cataplasmas e bandagens, injeção de substâncias osteogênicas (oleato de sódio), injeção intralesional de corticosteróide, cauterização (ponta de fogo), causticação (vesicatórios), escarificações, crioterapia, terapia por ondas de choque e drenagem de hematomas subperiostais. Esses tratamentos possuem graus variados de sucesso, não havendo na literatura estudos comparativos e controlados (BUCKINGHAM \& JEFFCOTT, 1990; BERTONE, 2002). Atualmente, em casos envolvendo reação periostal intensa e extensa e em casos de fraturas corticais, tem-se recorrido à técnica cirúrgica osteotixis (HANIE et al., 1992; BERTONE, 2002).

LARKIN \& DAVIES (1996) desenvolveram um índice radiográfico das proporções ósseas do MCIII, no intuito de prevenir a enfermidade. Concluíram que o repouso prolongado, em caso de periostite do MCIII, é contra-producente para a qualidade e resistência do osso em questão, posto que a falta de treinamento diminui a força e a massa esquelética (FORWOOD \& BURR, 1993).

Este trabalho propôs estudar um protocolo fisioterapêutico para o tratamento da periostite do MCIII em eqüinos, para oferecer uma opção terapêutica a mais para o combate dessa enfermidade.

\section{MATERIAL E MÉTODOS}

Foram tratados 24 ossos MCIII de 13 cavalos de corrida da raça PSI, nove machos e quatro fêmeas, com idade entre dois e três anos, em início de treinamento, sendo 21 casos classificados radiograficamente como periostite do MCIII subaguda ou crônica, devido à presença de reações osteoproliferativas sem fraturas corticais, e três classificados como fratura cortical, pois apresentavam imagem de fissura. 
Os animais permaneceram em repouso durante os primeiros dois dias de tratamento, iniciando exercício ao passo no terceiro dia, e trote seguido de retorno ao treinamento após a ausência da claudicação. $\mathrm{O}$ primeiro dia de tratamento sempre coincidiu com o início dos sinais clínicos e nenhum dos animais recebeu medicamento antiinflamatório.

O retorno ao treinamento compreendeu 1000 metros ao trote por uma semana, seguido por 2000 metros ao trote na segunda semana e 1500 metros ao trote associado a 500 metros em galope na terceira semana. A partir deste momento, cada animal foi submetido ao esquema de treinamento utilizado por seus treinadores, sendo informado aos autores os casos de recidiva da enfermidade.

\section{Exame físico}

O exame físico foi realizado diariamente, nos primeiros 10 dias, passando a ser em dias alternados até completar 30 dias de tratamento. Foi avaliada a presença e consistência de aumento de volume, postura antálgica, calor, dor local à palpação (1- leve, 2moderado, 3-severo), e analisou-se a dinâmica, classificando-se a claudicação de zero a cinco (STASHAK, 2002).

\section{Exame radiográfico}

Para a avaliação das alterações da superfície óssea, utilizou-se aparelho de raio-x portátila ${ }^{a}$ e foram realizadas três projeções radiográficas de cada MCIII a ser tratado: lateromedial, dorsolateral-palmaromedial oblíqua e dorsomedial-palmarolateral oblíqua, nos dias $1,14,21$ e 30.

\section{Exame ultra-sonográfico}

O exame ultra-sonográfico foi realizado nos dias 1, 7, 14, 21 e 30, para que fosse acompanhado o processo de reparação tecidual. Foi utilizado equipamento de ultra-som da marca Aloka ${ }^{\mathrm{b}}$, com transdutor linear multifreqüencial $(7,5 \mathrm{MHz})^{\mathrm{c}}$ acoplado a um adaptador de função. Foram adotados os seguintes critérios:

- avaliação da ecogenicidade da pele e do tecido subcutâneo;

- $\quad$ presença de irregularidade e de alteração de ecogenicidade do periósteo;

- avaliação do tendão extensor digital comum (espessura, forma, ecogenicidade e arquitetura).

\section{Protocolo fisioterapêutico}

Nos primeiros 10 dias, a fisioterapia foi realizada diariamente, sempre precedida do exame físico. A partir de então, as sessões e o exame físico passaram a ser em dias alternados, até completar 30 dias de tratamento.

Crioterapia - Realizada durante 20 minutos, quatro vezes ao dia, utilizando-se gelo em escamas. Esta técnica foi adotada somente nas primeiras 48 horas, a partir dos primeiros sinais clínicos observados.

Fototerapia e estimulação elétrica - A partir do terceiro dia, o tecido alvo foi exposto à fototerapia pulsátil ${ }^{\mathrm{d}}$ (50\%) por emissão de luz de comprimento de onda de $810 \mathrm{~nm}(\lambda=810 \mathrm{~nm})$. A aplicação teve duração de 20 minutos por dia, perfazendo uma dosagem de emissão de energia de $32 \mathrm{Jcm}^{-2}$.

No mesmo dia do início da fototerapia, a região foi submetida à eletroestimulação. $\mathrm{O}$ aparelho ${ }^{\mathrm{e}}$ utilizado emitia corrente alternada, a uma freqüência de duas a três ondas por segundo $(2 \mathrm{a} 3 \mathrm{~Hz})$, sendo que os eletrodos de silicone (6 cm de diâmetro) foram adaptados nas extremidades proximal e distal do MCIII.

Fonoterapia - A partir do quinto dia, foram acrescentados os efeitos do ultra-som ${ }^{\mathrm{f}}$ de $1 \mathrm{MHz}$, transdutor de $5 \mathrm{~cm}$ de diâmetro, pulsátil 20\%, na dosagem de $0,3 \mathrm{Wcm}^{-2}$, por aproximadamente seis minutos por dia.

\section{Análise estatística}

Os dados não paramétricos, grau de dor e de claudicação, foram analisados utilizando-se o teste de Friedman. Dados paramétricos foram submetidos à análise de variância (ANOVA). O contraste entre médias foi feito pela d.m.s. do teste de Tukey, em um nível de significância de $5 \%(a<0,05)$.

\section{RESULTADOS}

Por ocasião do exame físico do primeiro dia, constatou-se que todos os animais apresentavam aumento de volume regional, e que apenas dois deles (8\%) mostravam postura antálgica. Também foi observado que 15 dos 24 MCIII avaliados (62\%) apresentavam calor local e 22 (92\%), quadro de dor, sendo 10 de grau severo. Em relação ao grau de claudicação, observou-se que 15 animais (62\%) apresentaram claudicação no primeiro dia de avaliação, sendo oito de grau 4.

No decorrer do tratamento, o aumento de volume na região metacarpiana manteve-se com consistência firme, porém regrediu progressivamente em todos os casos. A postura antálgica não mais foi observada a partir do segundo dia. Após sete dias de tratamento, dois animais (8\%) ainda apresentavam calor local, 11 (46\%) mostravam dor, sete (29\%) apresentavam claudicação, e nenhum se mantinha em postura antálgica. No décimo quarto dia de tratamento, somente 
um animal (4\%) ainda apresentava calor local, quatro (17\%) mantinham dor local e cinco (21\%) apresentavam claudicação grau 1. Completados 20 dias de tratamento, não foi mais observado calor local, e as intensidades de dor e claudicação foram reduzidas a $8 \%$. Passados 30 dias de tratamento, somente um animal (4\%) apresentava dor leve e grau 1 de claudicação. Estatisticamente, houve diminuição do número de casos com presença de calor local no terceiro dia de tratamento, a redução no grau de dor ocorreu no sexto dia de tratamento e a redução no grau médio de claudicação no décimo dia $(\mathrm{P}<0,05)$.

A avaliação radiográfica inicial apresentou um ou mais calos subperiostais, ou seja, reações osteoproliferativas sem fraturas corticais em 21 casos, sendo que as lesões se encontravam principalmente na face dorsomedial (20/24) do terço médio (23/24) da diáfise. Em outros três casos foram observadas imagens de fissura cortical (Figura 1A).

O exame radiográfico mostrou maior homogeneidade na radiopacidade da reação osteoproliferativa, bem como seu adelgaçamento em todos os casos, no décimo quarto dia de tratamento. Também foi notada diminuição do volume de tecidos moles. Aos 21 dias de tratamento, foi observado início de espessamento da cortical óssea e continuidade do adelgaçamento antes verificado. Também se observou maior homogeneidade entre a lesão e a cortical óssea em relação à radiopacidade. O espessamento da cortical óssea foi nitidamente visto em todos os casos aos 30 dias de tratamento, sendo que, em alguns deles, já se notava o adelgaçamento da mesma. O aumento gradativo da radiopacidade no decorrer do experimento apresentou-se com superfície óssea lisa e de radioluscência homogênea (Figura 1B) em 20 casos, inicialmente classificados como subagudo ou crônico, e em um caso classificado como fratura cortical.

Dois dos três casos de fratura cortical ainda apresentaram, no último exame radiográfico, imagem de fissura, sutil e de difícil visualização. O acompanhamento do terceiro caso mostrou que a reparação óssea se completou em um mês, o retorno ao treinamento aconteceu neste mesmo tempo e a participação na primeira corrida um mês depois, ou seja, dois meses e meio a partir do início do protocolo.

$\mathrm{Na}$ análise qualitativa de todas as imagens obtidas ao exame ultra-sonográfico, não foram notadas alterações de ecogenicidade na pele e no subcutâneo que pudessem ser relacionadas a hematomas e outras coleções líquidas ou fibrose. Quanto à arquitetura, forma e ecogenicidade do tendão extensor digital comum não foram observadas alterações, e não houve alteração de sua espessura no decorrer do tratamento $(\mathrm{P}>0,05)$.
Na avaliação inicial do padrão ecográfico do periósteo, nas várias faces do osso MCIII, pôde-se observar, em todos os casos, irregularidades sob a forma de uma faixa paralela à superfície óssea preenchida por ecos curtos longitudinais, porém com menor definição que o habitual de seu limite contíguo ao tendão (Figura 2A). A evolução para a reparação desse tecido evidenciou-se pela regularização gradual dessa faixa até tornar-se contínua e lisa em seu limite contíguo ao tendão extensor, com definição clara de seu limite, e ainda pela redução da espessura e ecogenicidade (Figura 2B). Na maioria dos casos, não houve diminuição significativa da espessura do periósteo como resposta à terapia.

Houve seis casos (33\%) de recidiva durante treinamento para corridas, sendo que todos foram classificados, no início do tratamento, como periostite subaguda ou crônica.

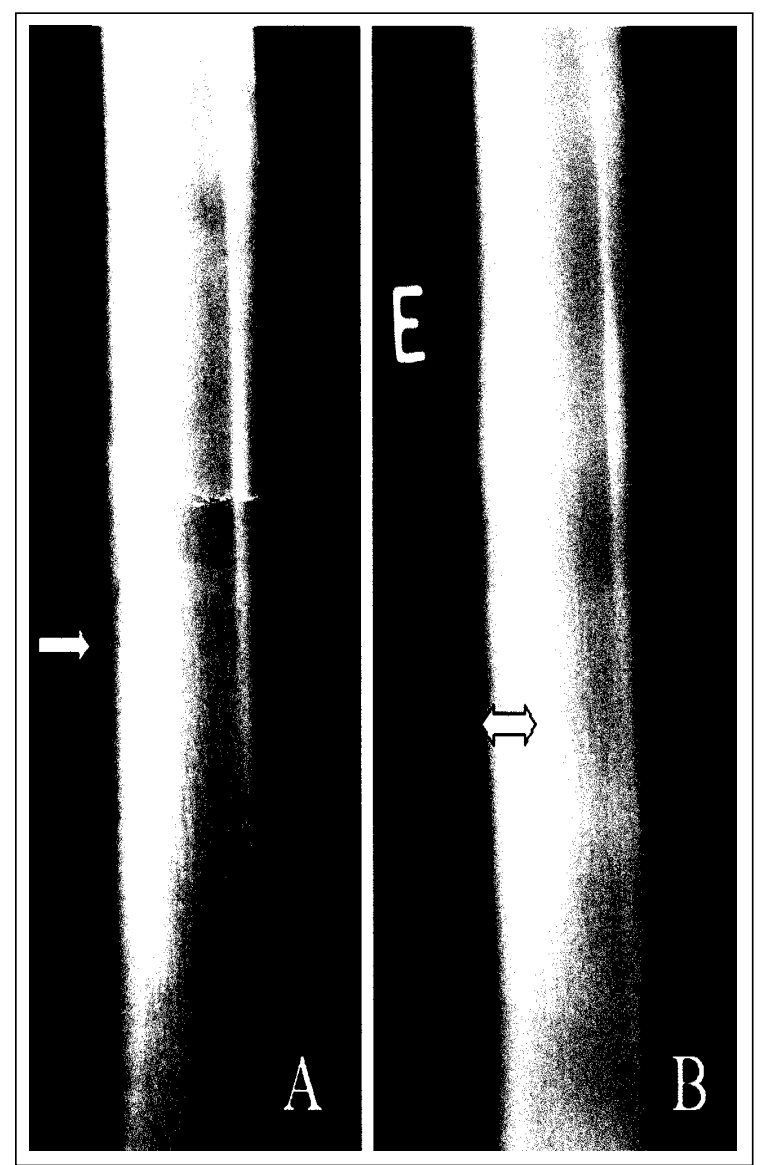

Figura 1 - Imagens radiográficas do osso MCIII de eqüino em posição dorsolateral-palmaromedial oblíqua. A: Reação osteoproliferativa na face dorsomedial no terço médio, com áreas de rarefação da cortical e linhas de fratura (seta) após 10 dias de tratamento. B: Espessamento homogêneo da cortical óssea (seta) do mesmo metacarpo, após 30 dias de tratamento. 


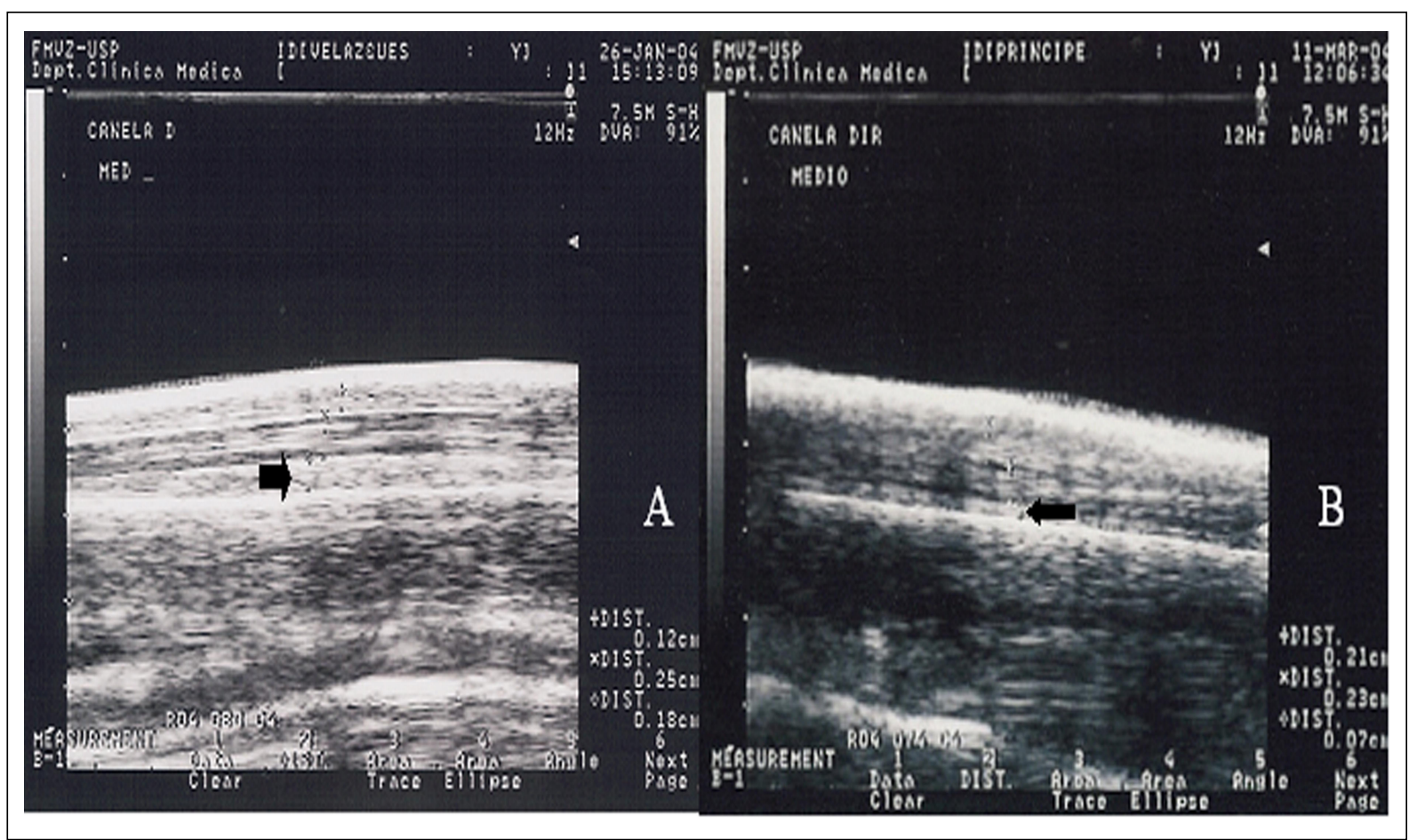

Figura 2 - Imagens ultra-sonográficas longitudinais da face dorsal do metacarpo de eqüino. A: periósteo espessado, com hiperecogenicidade anormal. B: periósteo com espessura normal, plano e liso no seu limite contíguo ao tendão extensor.

\section{DISCUSSÃO}

Vários autores (STASHAK, 1987; FORWOOD \& BURR, 1993; LARKIN \& DAVIES, 1996) consideraram contra-producente o repouso prolongado para a melhora da qualidade e resistência ósseas. A fisioterapia, favorecendo a diminuição dos sintomas, possibilitou a manutenção de atividade física durante o tratamento.

Pela avaliação da evolução clínica concernente ao grau de calor, observou-se que, após cinco dias de tratamento, metade dos animais não mais apresentava dor à palpação, e a outra metade mostrava somente grau leve de dor. Apesar da evidente regressão no grau de dor, observada no quarto dia de tratamento, este dado somente foi estatisticamente significativo no sexto dia $(\mathrm{P}<0,05)$. Do mesmo modo, houve redução do grau médio de claudicação no quinto dia, porém esta redução somente foi estatisticamente significativa a partir do décimo dia de tratamento $(\mathrm{P}<0,05)$.

Mesmo assim, as presenças de calor local e de dor à palpação mostraram tendência evidente de diminuição de intensidade e, concomitantemente, o grau de claudicação mostrou uma queda abrupta no decorrer da primeira semana de tratamento e continuou em declínio no decorrer do tratamento. Acredita-se então no efeito analgésico e antiinflamatório dos recursos fisioterapêuticos, pois nenhum medicamento antiinflamatório foi utilizado no decorrer do tratamento, e o repouso somente foi empregado nas primeiras 48 horas após a observação dos sintomas.

Ocorreu obviamente redução da intensidade do exercício, porém, segundo BERTONE (2002), somente as lesões agudas respondem unicamente ao repouso ou à redução da intensidade do exercício. Já as lesões subagudas ou crônicas necessitam geralmente de 110 dias de repouso para se resolverem, e o período de convalescença para fraturas corticais pode se prolongar de quatro a seis meses.

Utilizando-se o exame radiográfico, foi possível acompanhar a evolução e adelgaçamento das reações periostais e o espessamento homogêneo da cortical. Além disso, em alguns casos, foi possível a observação do adelgaçamento da cortical já aos 30 dias de tratamento, indicando finalização do processo de reparação. E seres humanos o tempo necessário para remodelagem óssea foi estabelecido como sendo em torno de três meses para crianças e de quatro a cinco meses para adultos. Nos cavalos, calcula-se que, em casos mais graves (periostite severa ou evidência de fratura), são necessárias de 12 a 24 semanas (três a seis meses) em esquema de repouso associado a exercício controlado (NORWOOD \& HAYNES, 1982). Neste 
experimento, radiograficamente, a superfície óssea tornou-se lisa e de radiopacidade homogênea em um mês para 21 casos.

Para resolução de fraturas corticais, é necessário um tempo de quatro a seis meses, segundo NORWOOD \& HAYNES (1982), ou mais, segundo HANIE et al. (1992). Utilizando-se a associação de métodos fisioterapêuticos, o tempo de evolução do processo de reparo da fratura mostrou-se significativamente diminuído em um dos casos, o que pode ser atribuído ao estímulo da atividade osteoblástica dos meios terapêuticos praticados no estudo e largamente pesquisada em diversos modelos animais (CARVALHO et al., 2002). Nos outros dois casos de fratura cortical, a imagem de fissura, sutil e de difícil visualização aos 30 dias de tratamento, também aponta para resultados benéficos do protocolo.

As imagens ultra-sonográficas tiveram como maior mérito permitir a observação da alteração das características do periósteo durante a evolução clínica da periostite do MCIII. As imagens ultra-sonográficas do periósteo mostraram seu espessamento, diferenças em quantidade e ecogenicidade dos ecos curtos, e presença ou ausência de irregularidade de sua camada fibrosa.

A manutenção da atividade mitótica (aumento de celularidade), do metabolismo tecidual e o incremento da circulação é suficiente para justificar a permanência do espessamento do periósteo no decorrer do tratamento, uma vez que os animais mantiveram-se em exercício controlado, permanecendo a necessidade de remodelagem óssea. O processo de reparação mostrou que o periósteo tem condição de fornecer elementos suficientes para a remodelagem e reparo ósseos, mas o caráter crônico da inflamação local compromete sua atuação, necessitando alguma forma de estímulo externo. Disto decorre a utilização da fisioterapia e de recursos invasivos agudizantes como a cauterização, a causticação e o osteotixis.

O efeito dos estímulos que levam à modulação da reação inflamatória com regressão dos sintomas é o mesmo que favorece a reparação por estimulação da osteogênese. Os recursos fisioterapêuticos, promovendo a melhora da circulação, e, portanto, do afluxo de oxigênio, nutrientes e água e ainda incrementando o metabolismo celular (LIPPIELLO \& SMALLEY, 1991), otimizam a osteogênese que se encontrava comprometida pelos efeitos deletérios da inflamação e pelo caráter crônico do processo. É importante salientar que um dos argumentos que justificam a prática da osteotixis, a saber, dar acesso da circulação e osteoblastos do endósteo ao local da fratura (COPELAN, 1979; STASHAK, 1987; HANIE et al., 1992), como forma de permitir a participação deste tecido na reparação, é o mesmo que indica o uso das técnicas fisioterapêuticas, ou seja, o aumento da circulação. Aparentemente, o periósteo adequadamente estimulado tem a circulação e metabolismo incrementados, condições estas suficientes para que ocorra o reparo das fraturas, evitando agressão aos tecidos. Além disso, segundo BANKS (1992), o endósteo e o periósteo não são anatomicamente independentes, dispensando procedimentos para estabelecer essa comunicação.

A associação de diferentes recursos, no entanto, impediu que cada estímulo per se fosse avaliado, exceção feita da crioterapia que foi aplicada isoladamente. Mesmo assim, esta foi associada ao repouso realizado nas primeiras 48 horas. Fato é que a associação de diminuição da intensidade do exercício com crioterapia, fototerapia, eletroestimulação e fonoterapia permitiu que os animais permanecessem em exercício durante o período de tratamento e retornassem ao esquema de treinamento para corridas em 30 dias. É importante frisar que a velocidade e a duração do exercício (NUNAMAKER et al., 1990; BOSTON \& NUNAMAKER, 2000), bem como a habilidade individual do animal em suportar exercícios de alta intensidade (DAVIES \& WATSON, 2005), estão diretamente correlacionadas com o aparecimento da enfermidade e com a porcentagem de recidivas. A impossibilidade de evitar a ocorrência de uma variável não prevista no protocolo, o tipo de trabalho imposto aos animais após o tratamento, interferiu no índice de recidivas da enfermidade. Infelizmente, as pressões para o pronto retorno dos potros à atividade normal, com vistas aos prêmios convidativos oferecidos para essa faixa de idade, dificultam a execução de estudos como este.

\section{CONCLUSÕES}

O protocolo fisioterapêutico proposto possibilita que os animais permaneçam em exercício durante o período de tratamento, permite a reparação tecidual em 30 dias e a continuidade de adaptação do periósteo, validando-o como método não invasivo de reabilitação nos casos de periostite do terceiro metacarpiano em eqüinos.

\section{AGRADECIMENTOS}

Os autores agradecem à Fundação de Pesquisa do Estado de São Paulo pelo apoio financeiro, e a todos os colegas do Jockey Club de São Paulo que de alguma forma contribuíram para este estudo.

Ciência Rural, v.36, n.3, mai-jun, 2006. 


\section{FONTES DE AQUISIÇÃO}

a MinXray, modelo HF100 - Estados Unidos da América.

b Aloka , modelo SSD-900 - Japão.

c Aloka, modelo UST-5710 - 7,5 MHz - Japão.

${ }^{d}$ Equi-Light - Estados Unidos da América.

e Soken - Estados Unidos da América

${ }^{\mathrm{f}} \mathrm{KLD}$, modelo portátil $-1 \mathrm{MHz}$ - Brasil.

\section{REFERÊNCIAS}

BANKS, W.J. Tecidos de sustentação. In: Histologia veterinária aplicada. 2.ed. São Paulo: Manole, 1992. Cap.9, p.137-166.

BECK, B.R. Tibial stress injuries: an aetiological review for the purposes of guiding management. Sports Medicine, v.26, n.4, p.265-279, 1998.

BERTONE, A. The metacarpus and metatarsus. In: STASHAK, T.S. Adam's lameness in horses. 5.ed. Philadelphia: Lippincott Williams \& Wilkins, 2002. Cap.8, p.800-863.

BOSTON, R.C.; NUNAMAKER, D.M. Gait and speed as exercise components of risk factors associated with onset of fatigue injury of the third metacarpal bone in 2-year-old throughbred racehorses. American Journal of Veterinary Research, v.61, n.6, p.602-608, 2000.

BUCKINGHAM, S.H.W.; JEFFCOTT, L.B. Shin soreness: a survey of thoroughbred trainers and racetrack veterinarians. Australian Equine Veterinary, v.8, n.4, p.148-153, 1990.

CARVALHO, D.C.L. et al. Tratamentos não farmacológicos na estimulação da osteogênese. Revista Saúde Pública, v.36, n.5, p.647-654, 2002.

COPELAN, R.W. Incidence, location and principles of treatment of stress fractures of the third metacarpal bone. In ANNUAL CONVENTION OF THE AMERICAN ASSOCIATION OF EQUINE PRACTITIONERS, 25., 1979, São Francisco, EUA. Proceedings... São Francisco: AAEP, 1979. p.159-162.

DAVIES, H.M.S. The effects of different exercise conditions on metacarpal bone strains in thoroughbred racehorses. Pferdekeilkunde, v.12, n.4, p.666-670, 1996.

DAVIES, H.M.; WATSON, K.M. Third metacarpal bone laterality asymmetry and midshaft dimensions in thoroughbred racehorses. Australian Veterinary Journal, v.83, n.4, p.224226, 2005.

FORWOOD, M.R.; BURR, D.B. Physical activity and bone mass: exercises in futility? Bone and Mineral, v.21, n.2, p.89-112, 1993.
HANIE, E.A. et al. A. Follow-up of 28 horses with third metacarpal unicortical stress fractures following treatment with osteotixis. Equine Veterinary Journal, n.11, Supplement, p.5-9, 1992.

IVERS, T. Cryotherapy. quine Practice, v.9, p.17-19, 1987.

LANG, D.C. Ultrasonic treatment of musculoskeletal conditions in horse, dog and cat. Veterinary Record, v.106, n.24, p.427431, 1980

LARKIN, N.C.; DAVIES, H.M.S. The application of a radiographic index to the prevention of dorsal metacarpal disease in thoroughbred racehorses. Pferdekeilkunde, v.12, n.4, p.595-598, 1996.

LIPPIELLO, L.; SMALLEY, L. Densitometric assessment of equine bone repair following pulsed ultrasound therapy. Equine Practice, v.13, n.4, p.4, 17, 20-22, 24, 1991.

MIKHAILOVA, L.N.; LANDA, V.A. Electron microscopic study of reparative osteogenesis following electric stimulation of bony tissue regeneration. Biulleten Eksperimentalnoi Biologii i Meditsiny, v.91, n.6, p.719-722, 1981.

MONTGOMERY, T.C. et al. Cryotherapy of dorsal metacarpal disease. Modern Veterinary Practice, v.62, n.3, p.219-20. 1981.

NORWOOD, G.L.; HAYNES, P.F. Dorsal metacarpal disease. In: MANSMANN, E.S. et al. Equine medicine and surgery. 3ed. Santa Barbara: American Veterinary, 1982. Cap.20, p.1110-1114.

NUNAMAKER, D.M. et al. Fatigue fractures in thoroughbred racehorse: relationships with age, peak bone strain, and training. Journal of Orthopaedic Research, v.8, n.4, p.604-611, 1990.

PORTER, $M$. The new equine sports therapy. Lexington: The Blood Horse, 1998. 205p.

REDDY, G.K. Photobiological basis and clinical role of low intensity lasers in biology and medicine. Journal of Clinical Laser Medicine and Surgery, v.22, n.2, p.141-150, 2004.

SPEIRS, V.C. Lameness: approaches to therapy and rehabilitation. In: HODGSON, D.R.; ROSE, R.J. The atletic horse. Philadelphia: Saunders, 1994. Cap.15, p.343-370.

STASHAK, T.S. Periostitis and fracture of the dorsal metacarpus (bucked shins, shin splints, and stress fracture). In:__. Adam's lameness in horses. 4.ed. Philadelphia:Lea \& Febiger, 1987. Cap.8, p.596-601

STASHAK, T.S. Examination for lameness. In:__. Adam's lameness in horses. 5.ed. Philadelphia: Lippincott Williams \& Wilkins, 2002. Cap.3, p.113-183. 